\title{
Cirurgia ortognática: abordagem psicossocial em pacientes Classe III de Angle submetidos à correção cirúrgica da deformidade dentofacial
}

\author{
Denise Nicodemo*, Max Domingues Pereira**, Lydia Masako Ferreira***
}

\begin{abstract}
Resumo
Objetivo: investigar aspectos psicossociais relacionados à mudança da aparência facial em 29 pacientes, de ambos os gêneros, com idades entre 17 e 46 anos, com indicação de tratamento cirúrgico, nos períodos pré-operatório (durante preparo ortodôntico) e pós-operatório (transcorridos 6 meses da intervenção cirúrgica). Métodos: utilizou-se questionários, aplicados na forma de entrevistas, com perguntas baseadas na proposta de Grossbart e Sarwer. Utilizou-se a técnica de análise de conteúdo, representando-se o motivo da procura pela correção cirúrgica em Categoria $1(\mathrm{Cl})$; as fantasias relacionadas aos resultados da correção cirúrgica (préoperatório) e realização (pós-operatório) em Categoria 2 (C2); e as expectativas e a satisfação quanto aos resultados da correção cirúrgica em Categoria 3 (C3). As respostas foram reagrupadas nas subcategorias: estética ( $\mathrm{SC}$ ), funcional (SC2), situações sociais (SC3), auto-estima (SC4) e profissional (SC5). Resultados: os resultados indicaram que os pacientes procuraram a correção cirúrgica por motivos funcionais $(34,5 \%)$, estéticos $(30,9 \%)$ e sociais $(29,1 \%)$; desejavam melhorar as situações sociais (40\%) e a estética (32\%), com realização destes desejos, depois da cirurgia. Quanto às expectativas, $49,4 \%$ dos pacientes esperavam melhorar o aspecto funcional, seguido da estética $(26,9 \%)$, situações sociais $(11,2 \%)$ e auto-estima $(6,7 \%)$. Em todos os aspectos, os pacientes ficaram muito satisfeitos pela melhora na dicção, na estética, na beleza e no retorno à vida sem discriminação. Conclusões: os pacientes procuraram a correção cirúrgica motivados a melhorar o aspecto funcional e a estética; fantasiavam melhorar as relações sociais e a aparência; esperavam, de forma realista, que a correção cirúrgica reparasse a função e a estética - objetivos propostos pela cirurgia ortognática.
\end{abstract}

Palavras-chave: Odontologia. Cirurgia plástica. Impacto psicossocial. Saúde bucal.

\footnotetext{
* Mestre e Doutora pelo Programa de Pós-Graduação em Cirurgia Plástica Reparadora - Universidade Federal de São Paulo - UNIFESP. Professora Responsável pela Disciplina de Psicologia Aplicada à Odontologia, Faculdade de Odontologia de São José dos Campos - Universidade Estadual Paulista "Júlio de Mesquita Filho" - UNESP.

** Professor Orientador do Programa de Pós-Graduação em Cirurgia Plástica Reparadora da Universidade Federal de São Paulo - UNIFESP.

*** Titular da Disciplina de Cirurgia Plástica e Coordenadora do Programa de Pós-Graduação em Cirurgia Plástica Reparadora da Universidade Federal de São Paulo - UNIFESP.
} 


\section{INTRODUÇÃO}

A cirurgia ortognática trata da correção cirúrgica das deformidades dentofaciais e a sua importância encontra-se não só na correção da oclusão, mas também da estética facial. Isto significa que os aspectos psicossociais estão diretamente relacionados a este tipo de tratamento, pois a aparência facial influencia a formação da imagem corporal, da identidade e da auto-estima.

A deformidade facial, com potencial psicológico e social destrutivo, causa impacto negativo, podendo influenciar não somente a autoconfiança dos pacientes, como também os relacionamentos externos, resultando em desvantagens sociais e psicológicas ${ }^{13,14,18,19,20,21}$. Os objetivos do paciente com deformidade dentofacial, relacionados à reparação, são também psicossociais e este pode expressar a expectativa de resolver suas dificuldades pessoais e sociais com a mudança física, ou seja, com a melhora de sua aparência pela correção cirúrgica.

Sendo assim, o processo de reparação da deformidade dentofacial, que envolve aspectos técnicos e psicossociais, necessita da cooperação do paciente e exige do profissional uma conduta integradora no trabalho em equipe multiprofissional ${ }^{1,22}$. A desconsideração de tais aspectos pode levar não só à insatisfação do paciente com os resultados do tratamento cirúrgico, mas até mesmo a problemas psicológicos pós-operatórios, como também à compreensão tardia, do cirurgião e equipe, que o insucesso (se ocorrido) pode ter sido resultado da falta de avaliação psicológica preliminar e falta de orientação apropriada ${ }^{3,4,25,27}$. Em pesquisa realizada por Power et al. ${ }^{26}$, na avaliação da percepção da qualidade de vida em 15 diferentes culturas, a auto-estima foi o item mais pontuado do domínio psicológico, denotando a importância da investigação destes fatores.

É crescente o número de estudos baseados na alegação de que a percepção do indivíduo acerca de si mesmo, do seu estado físico e emocional, é um indicador importante no delineamento do tratamento e que denota a inter-relação entre saúde bucal, saúde geral e qualidade de vida ${ }^{6,15}$. Considerando-se a influência dos aspectos psicossociais no tratamento das deformidades dentofaciais e o impacto que a cirurgia ortognática provoca, pela mudança da aparência, objetivou-se investigar aspectos psicossociais relacionados à mudança da aparência facial em pacientes Classe III de Angle, submetidos à cirurgia ortognática.

\section{MÉTODOS \\ Casuística}

Foram avaliados 29 pacientes, 13 do gênero masculino e 16 do gênero feminino, com idades entre 17 e 46 anos, apresentando má oclusão Classe III de Angle, com indicação de tratamento cirúrgico e que realizaram o preparo ortodôntico na Clínica de Ortodontia Corretiva do Curso de PósGraduação em Odontologia da Universidade Metodista do Estado de São Paulo (UMESP) - Brasil, durante 1 ano a 1 ano e 6 meses.

Após o preparo ortodôntico, os pacientes realizaram a cirurgia no Hospital São Paulo, no Setor de Cirurgia Craniomaxilofacial da Universidade Federal de São Paulo (UNIFESP) - Brasil.

\section{Descrição da amostra}

Dos 29 pacientes avaliados, 16 eram do gênero feminino $(55,1 \%)$ e 13 do masculino $(44,8 \%)$; a maioria solteira, estudante, com idades entre $17 \mathrm{e}$ 22 anos $(75,8 \%)$, tendo como escolaridade o ensino médio $(62 \%)$.

\section{Procedimentos}

Os pacientes foram avaliados em dois períodos: pré e pós-operatório. O pré-operatório referese ao período em que submeteram-se ao preparo ortodôntico, mais precisamente durante os últimos trinta dias que antecederam a cirurgia; e o pós-operatório depois de transcorridos seis meses da intervenção cirúrgica. A avaliação consistiu de questionários elaborados e aplicados, pelo pesquisador, na forma de entrevistas. Este procedimento foi adotado para os dois períodos, pré e pós-ope- 
ratórios e, em seguida, foi realizada a análise dos resultados.

\section{Questionários}

O questionário do período pré-operatório foi elaborado baseando-se nas perguntas propostas por Grossbart e Sarwer ${ }^{16}$. Constitui-se de 13 perguntas com respostas abertas, sobre a percepção que o paciente tem de sua deformidade; motivos, fantasias e expectativas com relação à correção cirúrgica; influências externas e conhecimento das etapas do processo.

O questionário do pós-operatório foi elaborado de forma que suas indagações (nove perguntas) respondessem às do primeiro questionário, contemplando respostas mistas, ou seja, fechadas (do tipo sim ou não) e abertas (para esclarecimentos da opção selecionada). Estas verificam se os pacientes ficaram ou não satisfeitos com os resultados da cirurgia; com realização ou não de suas fantasias e como perceberam as reações das pessoas. Quanto à satisfação das expectativas relacionadas aos resultados cirúrgicos, questionou-se o grau de realização das expectativas segundo a escala: 1 = muito; 2 = razoável ou médio; 3 = pouco e 4 = não satisfeito.

\section{Análise dos resultados}

Para análise das respostas dos questionários, utilizou-se a técnica de Análise de Conteúdo, do tipo categorial, para as questões 4,5 e 6 . Neste procedimento, realiza-se o desmembramento do texto em categorias, depois de uma leitura livre ou flutuante do material, segundo agrupamentos e reagrupamentos por significados ou aproximações semânticas ${ }^{1}$.

As respostas das questões 4, 5 e 6 foram agrupadas em categorias. Estas categorias foram chamadas de Categoria 1 (C1), Categoria 2 (C2) e Categoria 3 (C3) que tratam dos motivos pelos quais os pacientes procuraram a correção cirúrgica, das fantasias e expectativas quanto aos resultados, respectivamente. As respostas de cada uma destas categorias foram reagrupadas, em cinco subcategorias: estética ( $\mathrm{SCl}$ ), funcional (SC2), situações sociais (SC3), auto-estima (SC4) e profissional (SC5), considerando a aproximação semântica do conteúdo.

Utilizou-se da estatística descritiva, com a tabulação e análise da freqüência das respostas de todas as questões do questionário e, no caso das questões 4, 5 e 6, depois de realizada a técnica de análise de conteúdo, acima descrita.

\section{RESULTADOS}

A tabela 1 demonstra as respostas dos pacientes quando inquiridos sobre a maneira como definem a deformidade.

A tabela 2 apresenta os valores gerais das subcategorias estética, funcional, situações sociais e auto-estima, na Categoria 1 (Motivo da procura pela correção cirúrgica).

A tabela 3 apresenta os valores gerais das subcategorias das fantasias relacionadas aos resultados da correção cirúrgica (C2).

Os valores gerais das subcategorias estética, funcional, situações sociais, auto-estima e profissional, das expectativas realistas quanto aos resultados da correção cirúrgica (C3), são apresentados na tabela 4.

As respostas apontaram que em todos os aspectos os pacientes ficaram muito satisfeitos; para $75 \%$ a melhora foi maior do que esperavam, na dicção, na estética, na beleza, no impacto positivo e no retorno à vida sem discriminação.

\section{DISCUSSÃO}

\section{Amostra e objetos de investigação}

Dos pacientes que participaram do estudo, a maioria tem entre 17 e 22 anos de idade e é formada por indivíduos solteiros $(86,21 \%)$. Bennett e Phillips ${ }^{2}$, revisando os principais aspectos da qualidade de vida relacionada à saúde de pacientes com desarmonia dentofacial grave, salientaram que o número de pacientes que necessitam e desejam realizar este tipo de cirurgia aumentou 
Tabela 1 - Maneira como o paciente define a deformidade, em freqüências absoluta e relativa.

\begin{tabular}{|c|c|c|}
\hline definição & $\mathbf{n}$ & $\%$ \\
\hline $\begin{array}{l}\text { problema no maxilar, queixo desenvolvido } \\
\text { demais, prognatismo }\end{array}$ & 17 & 58,62 \\
\hline problema na mandíbula & 02 & 6,90 \\
\hline gengiva aparente & 01 & 3,45 \\
\hline $\begin{array}{c}\text { dores de cabeça, dificuldades na mastiga- } \\
\text { ção e no tratamento dentário }\end{array}$ & 03 & 10,33 \\
\hline queixo maior que dos outros & 01 & 3,45 \\
\hline problema de família & 02 & 6,90 \\
\hline $\begin{array}{c}\text { é uma coisa ruim, um incômodo, } \\
\text { provoca raiva }\end{array}$ & 02 & 6,90 \\
\hline não tem quem explique & 01 & 3,45 \\
\hline total & 29 & 100 \\
\hline
\end{tabular}

Tabela 2 - Categoria 1 - motivo da procura pela correção cirúrgica, por gênero.

\begin{tabular}{|c|c|c|c|c|c|c|}
\hline \multirow{2}{*}{$\begin{array}{c}\text { categoria } 1 \text { / } \\
\text { subcategorias }\end{array}$} & \multicolumn{2}{|c|}{ masculino } & \multicolumn{2}{|c|}{ feminino } & \multicolumn{2}{|c|}{ total } \\
\hline & n & $\%$ & $\mathbf{n}$ & $\%$ & n & $\%$ \\
\hline $\begin{array}{c}\text { subcategoria } 1 \\
\text { (estética) }\end{array}$ & 8 & 14,54 & 9 & 16,37 & 17 & 30,91 \\
\hline $\begin{array}{c}\text { subcategoria } 2 \\
\text { (funcional) }\end{array}$ & 7 & 12,72 & 12 & 21,82 & 19 & 34,54 \\
\hline $\begin{array}{c}\text { subcategoria } 3 \\
\text { (situações sociais) }\end{array}$ & 5 & 9,10 & 11 & 20,00 & 16 & 29,10 \\
\hline $\begin{array}{c}\text { subcategoria } 4 \\
\text { (auto-estima) }\end{array}$ & 2 & 3,64 & 1 & 1,81 & 3 & 5,45 \\
\hline $\begin{array}{c}\text { subcategoria } 5 \\
\text { (profissional) }\end{array}$ & 0 & 0 & 0 & 0 & 0 & 0 \\
\hline total de respostas & 22 & 40,00 & 33 & 60,00 & 55 & 100 \\
\hline
\end{tabular}

Tabela 3 - Categoria 2 - fantasias relacionadas aos resultados da correção cirúrgica (pré-operatório) por gênero e realização (pós-operatório).

\begin{tabular}{|c|c|c|c|c|c|c|c|c|}
\hline \multirow{3}{*}{$\begin{array}{c}\text { categoria } 2 / \\
\text { subcategorias }\end{array}$} & \multicolumn{6}{|c|}{ fantasias pré-operatório } & \multirow{2}{*}{\multicolumn{2}{|c|}{$\begin{array}{c}\text { realização pós-operatório } \\
\text { total }\end{array}$}} \\
\hline & \multicolumn{2}{|c|}{ masculino } & \multicolumn{2}{|c|}{ feminino } & \multicolumn{2}{|c|}{ total } & & \\
\hline & $\mathbf{n}$ & $\%$ & $\mathbf{n}$ & $\%$ & $\mathbf{n}$ & $\%$ & $\mathbf{n}$ & $\%$ \\
\hline subcategoria 1 (estética) & 11 & 14,66 & 13 & 17,34 & 24 & 32,00 & 23 & 30,66 \\
\hline subcategoria 2 (funcional) & 2 & 2,66 & 0 & 0 & 2 & 2,66 & 2 & 2,66 \\
\hline subcategoria 3 (situações sociais) & 15 & 20,00 & 15 & 20,00 & 30 & 40,00 & 26 & 34,66 \\
\hline subcategoria 4 (auto-estima) & 3 & 4,00 & 9 & 12,00 & 12 & 16,00 & 11 & 14,66 \\
\hline subcategoria 5 (profissional) & 4 & 5,34 & 3 & 4,00 & 7 & 9,34 & 2 & 2,66 \\
\hline total de respostas & 35 & 46,66 & 40 & 53,34 & 75 & 100 & 64 & 85,30 \\
\hline
\end{tabular}

Tabela 4 - Categoria 3 - expectativas quanto aos resultados da correção cirúrgica.

\begin{tabular}{|c|c|c|c|c|c|c|}
\hline \multirow{3}{*}{$\begin{array}{c}\text { categoria } 3 \text { / } \\
\text { subcategorias }\end{array}$} & \multicolumn{6}{|c|}{ expectativas } \\
\hline & \multicolumn{2}{|c|}{ masculino } & \multicolumn{2}{|c|}{ feminino } & \multicolumn{2}{|c|}{ total } \\
\hline & $\mathbf{n}$ & $\%$ & $\mathbf{n}$ & $\%$ & $\mathbf{n}$ & $\%$ \\
\hline subcategoria 1 (estética) & 10 & 11,23 & 14 & 75,74 & 24 & 26,97 \\
\hline subcategoria 2 (funcional) & 23 & 25,86 & 21 & 23,59 & 44 & 49,45 \\
\hline subcategoria 3 (situações sociais) & 2 & 2,24 & 8 & 8,99 & 10 & 11,23 \\
\hline subcategoria 4 (auto-estima) & 4 & 4,50 & 2 & 2,24 & 6 & 6,74 \\
\hline subcategoria 5 (profissional) & 3 & 3,37 & 2 & 2,24 & 5 & 5,61 \\
\hline total de respostas & 42 & 47,20 & 47 & 52,80 & 89 & 100 \\
\hline
\end{tabular}

Tabela 5 - Conhecimento sobre o procedimento cirúrgico.

\begin{tabular}{|c|c|c|c|c|c|c|c|c|}
\hline \multirow{2}{*}{ conhecimento } & \multicolumn{2}{|c|}{$\begin{array}{l}\text { gostaria de receber } \\
\text { mais informações }\end{array}$} & \multicolumn{2}{|c|}{$\begin{array}{c}\text { está satisfeito } \\
\text { com as informações }\end{array}$} & \multicolumn{2}{|c|}{$\begin{array}{l}\text { não quer saber } \\
\text { mais nada }\end{array}$} & \multicolumn{2}{|c|}{ total } \\
\hline & $\mathbf{n}$ & $\%$ & $\mathbf{n}$ & $\%$ & $\mathbf{n}$ & $\%$ & $\mathbf{n}$ & $\%$ \\
\hline sim, tem conhecimento & 8 & 27,58 & 6 & 20,69 & 3 & 10,34 & 17 & 58,63 \\
\hline não tem conhecimento & 12 & 41,37 & 0 & 0 & 0 & 0 & 12 & 41,37 \\
\hline total & 20 & 68,96 & 6 & 20,69 & 3 & 10,34 & 29 & 100 \\
\hline
\end{tabular}


e que eles são cada vez mais jovens, o que está evidenciado nesta amostra. Estes autores também salientaram que a avaliação feita pelo paciente é o indicador mais importante no tratamento, considerando que pode ocorrer insatisfação com os resultados cirúrgicos, mesmo que tecnicamente bem sucedidos. Um ponto que pode ser verificado, se não na totalidade mas na maioria das pesquisas de cirurgia ortognática, é que o conhecimento de aspectos motivacionais, psicológicos e sociais não pode ser negligenciado pela equipe de saúde, independentemente das formas de abordagem do assunto $^{5,11,17}$.

\section{Questionários}

Os trabalhos descritos na literatura, que abordam os instrumentos de avaliação do perfil psicossocial, assinalam a importância e complementaridade dos métodos qualitativo e quantitativo na pesquisa em saúde. Os trabalhos sobre aspectos psicológicos dos pacientes que se submetem à cirurgia ortognática são unânimes ao afirmar a importância do preparo psicológico antes da cirurgia, independentemente do método de investigação utilizado nas pesquisas: questionários e/ou testes padronizados. Neste preparo, o conhecimento dos motivos e expectativas para tal procedimento cirúrgico é imprescindível, pois é o momento em que informações e esclarecimentos serão transmitidos e expectativas irreais poderão ser trabalhadas pelo cirurgião e equipe, podendo se evitar insatisfação com resultados, mesmo que bem sucedidos tecnicamente.

Ao se pensar em conhecer os aspectos psicossociais, este estudo baseou-se nas questões sugeridas por Grossbart e Sarwer ${ }^{16}$. Embora estes autores não retratem especificamente a cirurgia ortognática, abordam a estética facial e sua abrangência na formação da personalidade e/ou das características pessoais como imagem corporal, autoconceito, autoconfiança nos relacionamentos sociais. Preconizam que se conheça o paciente antes de qualquer intervenção de natureza esté- tica, perguntando como ele define sua deformidade; qual o motivo por ter procurado por uma correção cirúrgica; o que espera obter (em nível real e fantasioso); porque acha que é o momento de realizar a cirurgia; se houve interferência de alguém para procurar e fazer a cirurgia; se a própria aparência faz lembrar de alguém que conhece. Em caso afirmativo, de quem; como acha que as pessoas importantes para ele irão reagir com o resultado, e as menos importantes; se o paciente consegue imaginar possíveis desvantagens com o procedimento a ser realizado.

A inclusão da questão sobre como o paciente define sua deformidade se deve, também, pela estreita relação que apresenta com os motivos que levam o paciente a procurar por tal cirurgia e suas expectativas, ou seja, o quê o paciente quer modificar, porquê e como ele acha que vai ficar depois da cirurgia. Da mesma forma, Peterson, Topazian $^{23}$; Epker, Fish ${ }^{8}$ e Cunningham ${ }^{5}$ incluíram, na entrevista pré-operatória, a questão de como o paciente define a sua deformidade, sob alegação de ser um indicador da melhor intervenção profissional.

As entrevistas do pós-operatório foram realizadas 6 meses após a intervenção. Considerou-se que, transcorrido este tempo, os pacientes estariam sem edema, teriam retomado as atividades rotineiras, profissionais e sociais e poderiam estar adaptados com a nova imagem. Acreditou-se que se a abordagem do pós-operatório fosse realizada mais precocemente, estes aspectos poderiam interferir na percepção do paciente acerca da sua aparência e, conseqüentemente, na satisfação com os resultados. Estudos futuros poderão investigar, especificamente, o processo de adaptação com a nova aparência, que não foi o objetivo deste estudo.

\section{Maneira como o paciente define a deformidade}

Verificou-se que as definições dos pacientes sobre a própria deformidade podem ser agrupadas em respostas técnicas e respostas pessoais. No pri- 
meiro caso, pode se observar o emprego de termos técnicos ou explicações mais próximas dos conceitos científicos, talvez pelo fato dos pacientes estarem sempre ouvindo os profissionais ou suas explicações e acabam apreendendo os conceitos, ou até mesmo por terem recorrido a informações científicas propriamente ditas. No segundo caso, de respostas mais pessoais ou mais informais, os pacientes definiram colocando suas percepções e dando seus significados.

Ao se procurar interpretar as definições dos pacientes além da terminologia empregada por eles, pode-se observar a representação da deformidade e o significado de tal aparência na vida de cada um deles. Uns dão maior valor ou se preocupam mais com a estética, outros mais com a saúde física e outros com o impacto negativo causado pela aparência, nas relações interpessoais.

\section{Motivo da procura pela correção cirúrgica}

A categoria 1, motivo da procura pela correção cirúrgica, apresentou maior concentração de respostas para a subcategoria funcional $(\mathrm{n}=19)$, seguida da estética $(n=17)$ e da subcategoria situações sociais $(n=16)$. Apenas 2 respostas foram registradas na subcategoria auto-estima: sentir-se bem ao se olhar no espelho $(n=1)$ e sentir-se melhor consigo mesmo $(n=2)$. Nenhuma resposta foi considerada para subcategoria profissional, nesta questão.

Estudos esclarecem os motivos da procura pela cirurgia ortognática, alguns concluem que são funcionais e estéticos, outros mais estéticos que funcionais. Garvill et al. ${ }^{13}$ e Wilmot et al. ${ }^{28}$ verificaram que os pacientes esperavam um resultado funcional e estético. Bertollini et al. ${ }^{3}$ discriminaram o motivo ou interesse primário como predominantemente estético.

Ao se analisar o motivo da procura pela cirurgia, verificou-se que as mulheres queriam melhorar tanto a função como as relações sociais, mais do que os homens. À semelhança, Phillips et al. ${ }^{24}$ demonstraram que as mulheres quiseram melhorar a função temporomandibular mais que os ho- mens, no entanto, diferiram quanto aos motivos sociais. Segundo os autores ${ }^{24}$, os homens tiveram, neste aspecto, maior interesse motivacional.

Grossbat e Sarwer ${ }^{16}$ consideraram que a maneira como o paciente define a sua deformidade está diretamente relacionada com o motivo pelo qual procurou o tratamento. Neste estudo, verificou-se que houve concordância entre estes pontos: as definições que os pacientes deram para as próprias deformidades relacionaram-se principalmente aos aspectos estético, funcional e social, assim como os motivos compreenderam as subcategorias estética, funcional e social.

\section{Fantasias relacionadas aos resultados da correção cirúrgica}

Quanto às fantasias, que representam o que se deseja num plano imaginário, sem fundamentação direta com a realidade, registrou-se maior número de respostas para as subcategorias: estética (32\%), situações sociais (40\%) e auto-estima (16\%). Os relatos dos pacientes compreenderam afirmações a respeito do desejo de ficarem mais bonitos e arrumarem namorado(a); mudarem a vida, ao se tornarem diferentes; viverem em paz, não tendo empecilhos e, por fim, serem felizes. As mulheres desejavam melhorar a auto-estima mais do que os homens e relataram que imaginaram que isso ocorreria mudando a forma de viver, sentindo realização pessoal, com o ego mais elevado. Em decorrência, pensou-se que as mulheres possam ter sofrido o impacto da aparência, antes da cirurgia, de forma mais intensa e negativa que os pacientes do gênero masculino, resultando em uma autoestima mais baixa. À semelhança, Garvill et al. ${ }^{13}$ concluíram que as mulheres sofreram a influência negativa da deformidade dentofacial mais intensamente que os homens.

No período pós-operatório, foi questionado se o paciente achava que as fantasias apontadas antes da cirurgia estavam se realizando ou não. Mesmo em se tratando de fantasias, onde se espera coisas irreais, muito subjetivas e, neste caso, distantes dos 
objetivos técnicos/cirúrgicos, verificou-se, contando com as respostas das entrevistas no período pós-operatório, que as fantasias que os pacientes tiveram, antes da cirurgia, estavam se realizando, segundo a percepção deles.

Peterson e Topazian ${ }^{23}$ se preocuparam em reconhecer a motivação dos pacientes submetidos à cirurgia ortognática, alegando ser este um aspecto importante para avaliar as expectativas. A idéia de que o paciente sempre espera alguma coisa quando se submete a um tratamento, principalmente relacionado à estética facial parece evidente, no entanto há de se pregar que o profissional envolvido no tratamento deve buscar o reconhecimento destes fatores. Cunningham et al. ${ }^{5}$, Grossbart e Sarwer $^{16}$ evidenciaram a necessidade de uma investigação detalhada dos aspectos psicossociais inerentes ao processo de cirurgia estética, abordando a interferência da aparência no desenvolvimento humano, na formação da auto-estima e imagem corporal.

\section{Expectativas quanto aos resultados da correção cirúrgica}

Quanto às expectativas reais, das 89 respostas levantadas, 44 se relacionaram com a subcategoria funcional $(49,45 \%) ; 34$ respostas compreenderam as outras duas categorias com maior pontuação: 24 para a estética e 10 para as situações sociais, com distribuição semelhante entre os gêneros.

A estética também é um objetivo deste tipo de intervenção, no entanto, remete a aspectos subjetivos da imagem corporal, da auto-estima e do conceito particular de beleza. MacGregor ${ }^{19}$ e Garvill et al. ${ }^{13}$ apontaram que a reparação tem um potencial benéfico, uma vez que a mudança da aparência influenciou positivamente tanto aspectos da vida pessoal como da vida social dos pacientes. Neste estudo, a estética foi também pontuada em nível de fantasia, só que neste aspecto registrou-se maior pontuação do que em motivos da procura pela cirurgia e igualdade de pontuação em expectativas com os resultados.
As situações sociais tiveram, nas fantasias, maior pontuação, embora registradas nas três categorias. Neste item, os pacientes expuseram desejos que não podem ser previstos pelo cirurgião e equipe, demonstrando coerência entre o porquê do tratamento e o que poderiam esperar dele. Ainda, seus desejos estavam em estreita dependência dos benefícios da nova aparência e melhora da função, e não do que o cirurgião e equipe poderiam dar pela intervenção técnica e cirúrgica. Considerando-se a distribuição das respostas entre as categorias, com pontuações diferentes nas subcategorias, pôde-se verificar que os pacientes estavam conscientes destes aspectos: sabiam o que tinham, o que queriam e o que poderiam esperar, e até sonhar.

\section{Conhecimento sobre o procedimento cirúrgico}

Dos 29 pacientes, 12 alegaram não ter conhecimento sobre o procedimento cirúrgico a ser realizado e gostariam de receber informações $(41,37 \%)$. Dos 8 pacientes que disseram ter o conhecimento $(27,58 \%), 6$ ficaram satisfeitos com as informações recebidas e 3 não queriam saber mais nada. As orientações e informações dadas aos pacientes podem favorecer relacionamentos adequados e positivos, aumentando a confiança, aspecto imprescindível no sucesso do tratamento. Verificou-se que os pacientes receberam informações, no entanto, a maior pontuação foi para aqueles que não receberam e, neste sentido, ressalta-se valer a pena um maior investimento neste aspecto, por parte dos profissionais envolvidos.

Flanary et al. ${ }^{12}$, Finlay et al. ${ }^{10}$, Cunningham et $a{ }^{7}{ }^{7}$, referindo-se à insatisfação, afirmaram que esta pode se relacionar à falta de preparação para a cirurgia ou de explicações insuficientes por parte do cirurgião e equipe. $\mathrm{O}$ preparo para a cirurgia deve ter também a finalidade de avaliar características de personalidade do paciente que podem ser prejudiciais, como problemas com a imagem corporal $5^{5}$. 
Acredita-se, assim como estes autores, que a área da saúde deve direcionar seus esforços e conhecimento na busca da melhor forma de relacionamento entre profissional e paciente. Considera-se, ainda, que a Biotecnologia poderá favorecer ainda mais este processo, caso não sejam priorizados apenas seus benefícios tecnológicos.

\section{CONCLUSÕES}

Concluiu-se que: 1) os pacientes procuraram a correção cirúrgica motivados a melhorar o aspecto funcional e a estética; 2) fantasiavam melhorar as relações sociais e a aparência; 3) esperavam, de forma realista, que a correção cirúrgica reparasse a função e a estética - objetivos propostos pela cirurgia ortognática.

\title{
Orthognathic surgery: psychosocial approach in Angle Class III patients submitted to correction surgery of facial deformity
}

\begin{abstract}
Aim: The purpose of this work was to investigate the psychosocial aspects related to facial changes in 29 patients, from both genres, between 17 and 46 years old, with indication of surgical treatment, in the preoperative (during orthodontic preparation) and in the postoperative (six months after the surgery) periods. Methods: Questionnaires, applied during interviews, with questions based on the proposal by Grossbart \& Sarwer were used. The technique of content analysis was used, in which the reason for choosing corrective surgery were represented in Category 1 (C1); fantasies related to the results of the corrective surgery (preoperative) and performance (postoperative) in Category 2 (C2); and expectations and satisfaction of the results of the corrective surgery in Category 3 (C3). The answers were grouped in the subcategories: aesthetics (SC1), functional (SC2), social situations (SC3), self-esteem (SC4) and professional (SC5). Results: the results showed that the patients looked for corrective surgery for functional reasons (34.5\%), aesthetics (30.9\%) and social (29.1\%); wished to improve his/her social situation (40\%) and the aesthetics (32\%), with the fulfillment of these wishes after the surgery. As for the expectations, $49.4 \%$ of the patients hoped to improve the functional aspect followed by aesthetics $(26.9 \%)$; social situations (11.2\%) and selfesteem (6.7\%). In all aspects the patients were very satisfied because of improvements in their diction, aesthetics, beauty, and the return to life without discrimination. Conclusions: It can be concluded that the patients looked for the corrective surgery with the motivation to improve their functional and aesthetics aspects, they fantasized to improve their social relations and appearance; and hoped, in a realistic way, that the corrective surgery would repaired the function and aesthetics - which are the objectives of the orthognathic surgery.
\end{abstract}

Key words: Plastic surgery. Psychosocial impact. Oral health.

\section{REFERÊNCIAS}

1. BARDIN, L. Análise de conteúdo. Lisboa: Edições 70, 1995.

2. BENNETT, M. E.; PHILLIPS, C. L. Assessment of health-related quality of life for patients with severe skeletal disharmony: a review of the issues. Int. J. Adult Orthod. Orthognath. Surg., Chicago, v. 14, p. 65-75, 1999.

3. BERTOLLINI, F.; RUSSO, V.; SANSEBASTIANO, G. Pre and post surgical psycho-emotional aspects of the orthognathic surgery patient. Int. J. Adult Orthod. Orthognath. Surg., Chicago, v. 15, p. $16-23,2000$.
4. CHEN, B.; ZHANG, Z. K.; WANG, X. Factors influencing postoperative satisfaction of orthognathic surgery patients. Int. J. Adult Orthod. Orthognath. Surg., Chicago, v. 17, p. 217-222, 2002.

5. CUNNINGHAM, S. J. The Psychology of facial appearance. Dent. Update, Guildford, v. 26, p. 438-443, 1999.

6. CUNNINGHAM, S. J.; GARRATT, A. M.; HUNT, N. P. Development of a condition-specific quality of life measure for patients with dentofacial deformity: II. Validity and responsiveness testing. Community Dent. Oral Epidemiol., Copenhagen, v. 30, p. 81-90, 2002. 
7. CUNNINGHAM, S. J.:HUNT, N. P. FEINMANN, C. Perceptions of outcome following orthognathic surgery. Br. J. Oral Maxillofac. Surg., Edinburgh, v. 34, p. 210-213, 1996.

8. EPKER, B.; FISH, L. C. Dentofacial deformities. In: _. Integrated orthodontic and surgical correction. St. Louis: Mosby, 1986. v. 1, p. 6-16

9. FERREIRA, L. M. Cirurgia plástica: uma abordagem antroposófica. Rev. SBCP, São Paulo, v. 19, p. 39-40, 2004

10. FINLAY, P. M.; ATKINSON, J. M.; MOOS, K. F. Orthognathic surgery: patient expectations; psychological profile and satis faction with outcome. Br. J. Oral Maxillofac. Surg., Edinburgh, v. 33, p. 9-14, 1995

11. FLANARY, C. M.; ALEXANDER, J. M. Patient responses to the orthognathic surgical experience: factors leading to dissatisfaction. J. Oral Maxillofac. Surg., Philadelphia, v. 41, p. 770-774, 1983.

12. FLANARY, C. M.; BARNWELL, G. M.; ALEXANDER, J. M. Patient perceptions of orthognathic surgery. Am. J. Orthod., St. Louis, v. 88, p. 137-145, 1985

13. GARVILL, J.; GARVILL, H.; KAHNBERG, K. E.; LUNDGREEN, S Psychological factors in orthognathic surgery. J. Craniomaxillofac. Surg., Edinburgh, v. 20, p. 28-33, 1992.

14. GERZANIC, L.; JAGSCH, R.; WATZKE, I. M. Psychologic implications of orthognathic surgery in patients with skeletal Class II or Class III malocclusion. Int. J. Adult Orthod. Orthognath. Surg., Chicago, v. 17, p. 75-81, 2002.

15. GIFT, H. C.; ATCHISON, K. A. Oral health, health, and health related quality of life. Med. Care, Hagerstown, v. 33, p. 57-77, 1995. Supplement.

16. GROSSBART, T. A.; SARWER, D. B. Cosmetic surgery: surgical tools: psychological goals. Semin. Cutan. Med. Surg., Philadelphia, v. 18, p. 101-111, 1999.

17. KIYAK, H. A.; WEST, R. A.; HOHL, T.; MCNEILL, R. W. The psychological impact of orthognathic surgery: a 9 month follow-up. Am. J. Orthod., St. Louis, v. 81, p. 404-412, 1982

18. LAZARIDOU-TERZOUDI, T.; KIYAK, H. A.; MOORE, R.; ATHANASIOU, A. E.; MELSEN, B. Long-term assessment of psycho- logical outcomes of orthognathic surgery. J. Oral Maxillofac. Surg., Philadelphia, v. 61, p. 545-552, 2003.

19. MacGREGOR, F. C. Facial disfigurement: problems and management of social interaction and implications for mental health. Aesthetic Plast. Surg., New York, v. 14, p. 249-257, 1990.

20. MOTEGI, E.; HATCH, J. P.; RUGH, J. D.; YAMAGUCHI, H. Health-related quality of life and psychosocial function 5 years after orthognathic surgery. Am. J. Orthod. Dentofacial Orthop., St. Louis, v. 124, p. 138-143, 2003.

21. NARDI, P.; ACOCELLA, A.; TEDESCO, A.; RISPOLI, A.; GIACOMELLI, E. Psychological aspects in orthognathic surgery: body image and quality of life in post surgical assessment. Minerva Stomatol., Torino, v. 52, p. 145-155, 2003.

22. NICODEMO, D.; RODE, S. M. Orientações psicológicas para o manejo clínico dos pacientes com indicação de prótese ocular. RPG Rev. Pós Grad., São Paulo, v. 9, p. 224-231, 2002.

23. PETERSON, L. J.; TOPAZIAN, R. G. Psychological consideration in corrective maxillary and midfacial surgery. J. Oral Surg. Chicago, v. 34, p. 157-164,1976

24. PHILLIPS, C.; BRODER, H. L.; BEBBETT, M. C. Dentofacial disharmony: motivations for seeking treatment. Int. J. Adult Orthod. Orthognath. Surg., Chicago, v. 12, p. 7-15, 1997.

25. POGREL, M. A.; SCOTT, P. Is it possible to identify the psychologically "bad risk" orthognathic surgery patient preoperatively? Int. J. Adult Orthod. Orthognath. Surg., Chicago, v. 9 p. 105-110, 1994

26. POWER, M.; HARPER, A.; BULLINGER, M. The World Health Organization WHOQOL-100: tests of the universality of quality of life in 15 different cultural groups worldwide. Health Psychol., Washington, D. C, v. 18, p. 495-505, 1999

27. SIOW, K. K.; ONG, S. T.; LIAN, C. B.; NGEOW, W. C. Satisfaction of orthognathic surgical patients in a Malaysian population. J. Oral Sci., Tokyo, v. 44, p. 165-171, 2002

28. WILMOT, J. J.; BARBER, H. D.; CHOU, D. G.; VIG, K. W. L. Associations between severity of dentofacial deformity and motivation for orthodontic-orthognathic surgery treatment. Angle Orthod., Appleton, v. 63, p. 283-288, 1993.
Endereço para correspondência

Denise Nicodemo

Av. Engenheiro Francisco José Longo, 777 - Jardim São Dimas

CEP: 12.244-000 - São José dos Campos/SP

E-mail: denise@fosjc.unesp.br 